\title{
SUBSERIES CONVERGENCE IN SPACES WITH A SCHAUDER BASIS
}

\author{
CHARLES SWARTZ
}

(Communicated by Palle E. T. Jorgensen)

\begin{abstract}
Let $E$ be a Hausdorff topological vector space having a Schauder basis $\left\{b_{i}\right\}$ and coordinate functionals $\left\{f_{i}\right\}$. Let $\sigma(E, F)$ be the weak topology on $E$ induced by $F=\left\{f_{i}: i \in \mathbf{N}\right\}$. We show that if a series in $E$ is subseries convergent with respect to $\sigma(E, F)$, then it is subseries convergent with respect to the original topology of $E$.
\end{abstract}

In [S] Stiles established what seems to be the first version of the Orlicz-Pettis Theorem for nonlocal spaces. Indeed, Kalton remarks in [K1] that Stiles's result motivated his far-reaching generalizations of the Orlicz-Pettis Theorem for series in a topological group [K2]. Stiles's version of the Orlicz-Pettis Theorem is for $F$-spaces with a Schauder basis, and his proof uses the metric properties of the space. Other proofs of Stiles's result have been given in [B] and [Sw1], and both of these proofs also rely heavily on the metric. Kalton has also given a substantial generalization of Stiles's result in the Corollary to Theorem 3 in [K2]; this generalization is also for metric linear spaces. In this note we will give a proof of Stiles's Orlicz-Pettis Theorem for arbitrary Hausdorff topological vector spaces which have a Schauder basis. Our proof uses a very interesting result of Antosik on the convergence of double series [A].

Throughout this note let $E$ be a Hausdorff topological vector space with a Schauder basis $\left\{b_{i}\right\}$ and coordinate functionals $\left\{f_{i}\right\}$. Let $F=\left\{f_{i}: i \in \mathbf{N}\right\}$, and let $\sigma(E, F)$ be the weak topology on $E$ from the duality between $E$ and $F$. If $\tau$ is any vector topology on $E$, a series $\sum x_{j}$ in $E$ is said to be $\tau$ subseries convergent if for every subsequence $\left\{x_{n_{j}}\right\}$ of $\left\{x_{j}\right\}$ the subseries $\sum x_{n_{j}}$ is $\tau$ convergent in $E$. We show that every series $\sum x_{j}$ in $E$ which is $\sigma(E, F)$ subseries convergent is subseries convergent in the original topology of $E$.

For the proof of our result we use the following theorem of Antosik on iterated double series $[\mathrm{A}]$.

Theorem 1 (Antosik). Let $x_{i j} \in E$ for $i, j \in \mathbf{N}$. If for every increasing sequence of positive integers $\left\{n_{j}\right\}$ the iterated series $\sum_{i=1}^{\infty} \sum_{j=1}^{\infty} x_{i n_{j}}$ converges, then the double series $\sum_{i, j} x_{i j}$ converges. In particular, the iterated series $\sum_{j=1}^{\infty} \sum_{i=1}^{\infty} x_{i j}$ converges and equals $\sum_{i=1}^{\infty} \sum_{j=1}^{\infty} x_{i j}$.

Received by the editors February 2, 1993 and, in revised form, May 7, 1993.

1991 Mathematics Subject Classification. Primary 46A35. 
Antosik proved Theorem 1 for series in a space equipped with a sequential convergence structure satisfying certain convergence properties [A, Theorem 3]. The theorem was established for series in an Abelian topological group in [Sw2]; the proof used only the Hahn-Schur Theorem for groups [AS, 8.1].

We now state and prove our generalization of Stiles's Orlicz-Pettis Theorem.

Theorem 2. If $\sum x_{j}$ is $\sigma(E, F)$ subseries convergent in $E$, then $\sum x_{j}$ is subseries convergent in the original topology of $E$.

Proof. We use Antosik's Theorem to show the convergence (in the original topology of $E$ ) of the double series $\sum_{i, j}\left\langle f_{i}, x_{j}\right\rangle b_{i}$. Let $\left\{n_{j}\right\}$ be an increasing sequence of positive integers, and let $x=\sum_{j=1}^{\infty} x_{n_{j}}$ be the $\sigma(E, F)$ sum of this series. For each $i, \sum_{j=1}^{\infty}\left\langle f_{i}, x_{n_{j}}\right\rangle b_{i}$ converges in $E$ to $\left\langle f_{i}, x\right\rangle b_{i}$ since $\sum_{j=1}^{\infty}\left\langle f_{i}, x_{n_{j}}\right\rangle=\left\langle f_{i}, x\right\rangle$. Then

$$
\sum_{i=1}^{\infty} \sum_{j=1}^{\infty}\left\langle f_{i}, x_{n_{j}}\right\rangle b_{i}=\sum_{i=1}^{\infty}\left\langle f_{i}, x\right\rangle b_{i}=x .
$$

By Theorem 1,

$$
\sum_{j=1}^{\infty} \sum_{i=1}^{\infty}\left\langle f_{i}, x_{j}\right\rangle b_{i}=\sum_{j=1}^{\infty} x_{j}
$$

converges in the original topology of $E$. Since the same argument can be applied to any subseries of $\sum x_{j}$, the result follows.

The use of Antosik's Interchange Theorem in the proof of Theorem 2 removes both the metrizability and completeness assumptions from Stiles's Theorem [S]. Note that we did not assume the continuity of the coordinate functionals $\left\{f_{i}\right\}$ in our proof, so the topology $\sigma(E, F)$ and the original topology of $E$ may not even be comparable.

The proof of Theorem 2 covers even more general situations than topological vector spaces with Schauder bases. We indicate an example of such an application.

Let $(G, \tau)$ be an abelian Hausdorff topological group, and assume there exists a sequence of homomorphisms $P_{i}: G \rightarrow G$ such that $\sum_{i=1}^{\infty} P_{i} x=x$ (convergence in $\tau)$ for each $x \in G$. In the case of $E$ above, $P_{i} x=\left\langle f_{i}, x\right\rangle b_{i}$ is an example; if $G$ is a vector space and each $P_{i}$ is a projection, then a sequence $\left\{P_{i}\right\}$ satisfying the condition above is called a Schauder decomposition. Antosik's Interchange Theorem is valid for group-valued series [Sw2], and the proof of Theorem 2 gives

Theorem 3. Let $\sigma$ be a Hausdorff group topology on $G$, and assume that each $P_{i}$ is $\sigma-\tau$ continuous. If $\sum x_{i}$ is $\sigma$ subseries convergent, then $\sum x_{i}$ is $\tau$ subseries convergent.

In Theorem 2, $P_{i} x=\left\langle f_{i}, x\right\rangle b_{i}$ is $\sigma(E, F)-\tau$ continuous, where $\tau$ is the original topology of $E$, so Theorem 2 is an immediate corollary of Theorem 3.

\section{REFERENCES}

[A] P. Antosik, On interchange of limits, Generalized Functions, Convergence Structures and their Applications, Plenum Press, New York, 1988, pp. 367-374.

[AS] P. Antosik and C. Swartz, Matrix methods in analysis, Springer-Verlag, Heidelberg, 1985. 
[B] B. Basit, On a theorem of Gelfand and a new proof of the Orlicz-Pettis Theorem, Rend. Istit. Mat. Univ. Trieste 18 (1986), 156-162.

[K1] N. J. Kalton, The Orlicz-Pettis Theorem, Contemp. Math., vol. 2, Amer. Math. Soc., Providence, RI, 1980, pp. 91-100.

[K2] - Subseries convergence in topological groups and vector measures, Israel J. Math. 10 (1971), 402-412.

[S] W. J. Stiles, On subseries convergence in F-spaces, Israel J. Math. 8 (1970), 53-56.

[Sw1] C. Swartz, A generalization of Stiles' Orlicz-Pettis Theorem, Rend. Istit. Mat. Univ. Trieste 20 (1988), 109-112.

[Sw2] _ _ Iterated series and the Hellinger-Toeplitz theorem, Publ. Mat. 36 (1992), 167-173.

Department of Mathematical Sciences, New Mexico State University, Las Cruces, New MeXico 88003

E-mail address: cswartz@nmsu.edu 